\title{
THE CYCLOTOMIC NUMBERS OF ORDER SEVEN ${ }^{1}$
}

\author{
PHILIP A. LEONARD AND KENNETH S. WILLIAMS
}

ABSTRACT. The cyclotomic numbers of order seven are given in terms of the solutions of a certain system of three quadratic diophantine equations. This is analogous to L. E. Dickson's evaluation of the cyclotomic numbers of order five, and is a convenient approach for applications to the theory of power residues.

1. Introduction. Let $g$ be a primitive root of an odd prime $p$. Let $e>1$ be a divisior of $p-1$ and write $p-1=e f$. The cyclotomic number $(h, k)=(h, k)_{e}$ is defined to be the number of solutions $s, t$ of the trinomial congruence

$$
g^{e s+h}+1 \equiv g^{e t+k}(\bmod p), \quad 0 \leq s, t \leq f-1 .
$$

A central problem in the theory of cyclotorny is to obtain formulae for the numbers $(h, k)$. The cases $e=2,3,4,5,6,8,9,10,12,14,15,16,18$ and 20 have been treated by several authors, beginning with L. E. Dickson [2][4], with fuller treatments due to Emma Lehmer ([6], $e=8)$, A. L. Whiteman ([13]-[15], $e=10,12,16)$, J. B. Muskat ([9], $e=14$ ), L. Baumert and H. Fredricksen ([1], $e=9,18)$, and Muskat and Whiteman ([10], $e=20)$.

When $e=7$ the cyclotomic numbers can be given in terms of certain Dickson-Hurwitz sums using the work of Muskat [9, Theorem 1] or a theorem of Whiteman [15, Theorem 1]. In this paper we obtain these cyclotomic numbers in terms of the solutions of a certain triple of diophantine equations, analogous to the expressions for the cyclotomic numbers of order 5 in terms of the solutions of a pair of diophantine equations (see for example [15, p. 101]). This formulation is often useful in applications (see $\$ 3$ ). We make use of the following recent result of the authors [7, Theorems 2 and 3]. If $p \equiv 1(\bmod 7)$ then there are exactly six integral simultaneous solutions of

Received by the editors July 15, 1973 and, in revised form, May 6, 1974.

AMS (MOS) subject classifications (1970). Primary 12C20; Secondary 10A15, 10B05, 10G05.

Key words and phrases. Cyclotomic numbers, Jacobi sums, Dickson-Hurwitz sums.

1 This research was supported by a grant (no. A7233) from the National Research Council of Canada. 
the triple of diophantine equations

$$
\begin{aligned}
72 p & =2 x_{1}^{2}+42\left(x_{2}^{2}+x_{3}^{2}+x_{4}^{2}\right)+343\left(x_{5}^{2}+3 x_{6}^{2}\right) \\
12 x_{2}^{2} & -12 x_{4}^{2}+147 x_{5}^{2}-441 x_{6}^{2}+56 x_{1} x_{6} \\
& +24 x_{2} x_{3}-24 x_{2} x_{4}+48 x_{3} x_{4}+98 x_{5} x_{6}=0 \\
12 x_{3}^{2} & -12 x_{4}^{2}+49 x_{5}^{2}-147 x_{6}^{2}+28 x_{1} x_{5} \\
& +28 x_{1} x_{6}+48 x_{2} x_{3}+24 x_{2} x_{4}+24 x_{3} x_{4}+490 x_{5} x_{6}=0
\end{aligned}
$$

satisfying $x_{1} \equiv 1(\bmod 7)$, distinct from the two "trivial" solutions $(-6 t, \pm 2 u, \pm 2 u, \mp 2 u, 0,0)$, where $t$ is given uniquely and $u$ is given ambiguously by

$$
p=t^{2}+7 u^{2}, \quad t \equiv 1(\bmod 7) .
$$

If $\left(x_{1}, x_{2}, x_{3}, x_{4}, x_{5}, x_{6}\right)$ is a nontrivial solution with $x_{1} \equiv 1(\bmod 7)$ then two others are given by $\left(x_{1},-x_{3}, x_{4}, x_{2}, 1 / 2\left(-x_{5}-3 x_{6}\right), 1 / 2\left(x_{5}-x_{6}\right)\right)$ and $\left(x_{1},-x_{4}, x_{2},-x_{3}, 1 / 2\left(-x_{5}+3 x_{6}\right), 1 / 2\left(-x_{5}-x_{6}\right)\right)$. Each of the other three can be obtained from one given above by changing the signs of $x_{2}, x_{3}, x_{4}$. It is surprising to us that this result, which parallels a similar result (see for example $[2,1$, Theorem 8$])$ for $p \equiv 1(\bmod 5)$, and which is implicit in the work of Dickson [2], [3], does not appear in the literature. See [5] and [11, p. 128] for comments related to $p \equiv 1(\bmod 7)$.

2. Calculation of the cyclotomic numbers of order 7 . The numbers $(h, k)$ satisfy the following well-known relations $[11$, p. 25]:

$$
\begin{aligned}
& (b, k)=(b+a e, k+b e) \text { for any integers } a \text { and } b, \\
& (b, k)=(k, b) \text { if } f \text { is even, } \\
& (b, k)=(e-b, k-b) .
\end{aligned}
$$

With $e=7$ the formulae (2.1), (2.2), (2.3) yield the matrix

$$
\left[\begin{array}{lllllll}
A & B & C & D & E & F & G \\
B & G & H & I & J & K & H \\
C & H & F & K & L & L & I \\
D & I & K & E & J & L & J \\
E & J & L & J & D & I & K \\
F & K & L & L & I & C & H \\
G & H & I & J & K & H & B
\end{array}\right]
$$


in which the letter in the $h$ th row and $k$ th column, $h, k=0,1,2, \cdots, 6$, represents the value of $(h, k)$. Thus the evaluation of the $e^{2}=49$ cyclotomic numbers of order 7 reduces to the determination of the 12 quantities $A, B, C, D, E, F, G, H, I, J, K, L$. (2.4) has been given by Whiteman [12, p. 63].

Let $g$ be any primitive root of the prime $p \equiv 1(\bmod 7)$ and set $\zeta=$ $\exp (2 \pi i / 7)$. For any integers $m$ and $n$ we define the Jacobi sum $J(m, n)$ by

$$
J(m, n)=\sum_{x, y=1 ; x+y \equiv 1(\bmod p)}^{p-1} \zeta^{m \text { ind }_{g} x+n \text { ind }_{g} y}
$$

where ind ${ }_{g} x$ denotes the unique integer $k$ such that $x \equiv g^{k}(\bmod p), 0 \leq$ $k \leq p-2$. It was shown in [7] that

$$
J(1,1)=\sum_{i=1}^{6} c_{i} \zeta^{i}
$$

the integers $c_{1}, \cdots, c_{6}$ being given by

$$
\begin{array}{rlrl}
12 c_{1} & =-2 x_{1}+6 x_{2}+7 x_{5}+21 x_{6}, & 12 c_{4} & =-2 x_{1}-6 x_{4}-14 x_{5}, \\
\text { (2.6) } 12 c_{2} & =-2 x_{1}+6 x_{3}+7 x_{5}-21 x_{6}, & 12 c_{5} & =-2 x_{1}-6 x_{3}+7 x_{5}-21 x_{6}, \\
12 c_{3} & =-2 x_{1}+6 x_{4}-14 x_{5}, & 12 c_{6}=-2 x_{1}-6 x_{2}+7 x_{5}+21 x_{6},
\end{array}
$$

where $\left(x_{1}, x_{2}, x_{3}, x_{4}, x_{5}, x_{6}\right)$ is a nontrivial solution of (1.1)-(1.3) satisfying $x_{1} \equiv 1(\bmod 7)$, and

$$
J(1,2)=-t+u \sqrt{-7}
$$

where the integers $t$ and $u$ satisfy $p=t^{2}+7 u^{2}, t \equiv 1(\bmod 7)$.

The Dickson-Hurwitz sums of order 7 are defined by

$$
J(1, j)=\sum_{i=0}^{6} B(i, j) \zeta^{i} \quad(j=0,1, \cdots, 6),
$$

and

$$
\sum_{i=0}^{6} B(i, j)=p-2
$$

They have the following properties (see for example [15, p. 97]):

$$
B(i, j)=B(i, 6-j)
$$

$$
B(i, 0)= \begin{cases}f-1 & \text { if } i=0 \\ f & \text { if } 1 \leq i \leq 6\end{cases}
$$




$$
B(i, j)=B(i \bar{j}, \bar{j}) \quad \text { if } j \neq 0 \text { and } j \bar{j} \equiv 1(\bmod 7) \text {. }
$$

Since $\sum_{i=1}^{6} c_{i}=-x_{1}$ by (2.6), (2.8) and (2.9) we obtain for $i=1,2, \cdots, 6$,

$$
B(i, 1)=c_{i}+B(0,1)=c_{i}+\left(p-2+x_{1}\right) / 7 .
$$

Also as $-1=\zeta+\zeta^{2}+\zeta^{3}+\zeta^{4}+\zeta^{5}+\zeta^{6}$ and $\sqrt{-7}=\zeta+\zeta^{2}-\zeta^{3}+\zeta^{4}-$ $\zeta^{5}-\zeta^{6}$, we obtain from (1.5), (2.7), (2.8), (2.9)

$$
\begin{aligned}
& 7 B(0,2)=-6 t+p-2, \\
& 7 B(1,2)=7 B(2,2)=7 B(4,2)=t+7 u+p-2, \\
& 7 B(3,2)=7 B(5,2)=7 B(6,2)=t-7 u+p-2 .
\end{aligned}
$$

Equation (2.14) is due to Muskat [9, p. 270]. Whiteman [15, Theorem 1] has shown that

$$
7(b, k)=\sum_{v=0}^{6} B(v b+k, v)-6 f+ \begin{cases}1 & \text { if } 7 \nmid h, \\ 0 & \text { if } 7 \mid h .\end{cases}
$$

Using this together with (2.6), (2.10), (2.11), (2.12), (2.13) and (2.14) we obtain the cyclotomic numbers in terms of $t, u, x_{1}, \ldots, x_{6}$. In applying these expressions given in the Theorem below we must indicate how the sign of $u$ is to be chosen given a nontrivial solution $\left(x_{1}, \ldots, x_{6}\right)$ of (1.2)(1.4) satisfying $x_{1} \equiv 1(\bmod 7)$. If $7 \nmid u$ this is easy as we see from the Theorem that $7(B-G)=4 u+2 x_{2}-x_{3}$, so we need only choose $u$ such that

$$
u \equiv 3 x_{2}+2 x_{3}(\bmod 7)
$$

If however $7 \mid u$ it appears to be necessary to use (2.5), (2.6), (2.7) and the identity

$$
p J(1,2)=J(1,1) J(2,2) J(4,4) .
$$

Thus, for example, when $p=379$ a nontrivial solution of (1.2)-(1.4) with $x_{1} \equiv 1(\bmod 7)$ is given by

$$
x_{1}=-13, \quad x_{2}=10, \quad x_{3}=13, \quad x_{4}=-12, \quad x_{5}=-5, \quad x_{6}=1,
$$

and so by (2.6) we have

$$
c_{1}=6, \quad c_{2}=4, \quad c_{3}=2, \quad c_{4}=14, \quad c_{5}=-9, \quad c_{6}=-4 .
$$

Using these values in $(2.5)$ and computing $J(1,1) J(2,2) J(4,4)$ we obtain from (2.7) and (2.16) that $t=-6, u=-7$. 
Theorem. Let $p$ be a prime $\equiv 1(\bmod 7)$. If $\left(x_{1}, \cdots, x_{6}\right)$ is any nontrivial solution of (1.2)-(1.4) with $x_{1} \equiv 1(\bmod 7)$ and $(t, u)$ is the solution of (1.5) with $t \equiv 1(\bmod 7)$ and $u$ given by (2.15) or by (2.16) as indicated above, then for some primitive root $g(\bmod p)$ the cyclotomic numbers of order 7 are given by (2.4) and

$$
\begin{aligned}
& 49 A=p-20-12 t+3 x_{1}, \\
& 588 B=12 p-72+24 t+168 u-6 x_{1}+84 x_{2}-42 x_{3}+147 x_{4}+147 x_{6}, \\
& 588 C=12 p-72+24 t+168 u-6 x_{1}+84 x_{3}+42 x_{4}-294 x_{6}, \\
& 588 D=12 p-72+24 t-168 u-6 x_{1}+42 x_{2}+84 x_{4}-147 x_{5}+147 x_{6}, \\
& 588 E=12 p-72+24 t+168 u-6 x_{1}-42 x_{2}-84 x_{4}-147 x_{5}+147 x_{6}, \\
& 588 F=12 p-72+24 t-168 u-6 x_{1}-84 x_{3}-42 x_{4}-294 x_{6}, \\
& 588 G=12 p-72+24 t-168 u-6 x_{1}-84 x_{2}+42 x_{3}+147 x_{5}+147 x_{6}, \\
& 588 H=12 p+12+24 t+8 x_{1}-196 x_{5}, \\
& 588 I=12 p+12-60 t-84 u-6 x_{1}+42 x_{2}+42 x_{3}-42 x_{4}, \\
& 588 \mathrm{~J}=12 p+12+24 t+8 x_{1}+98 x_{5}-294 x_{6}, \\
& 588 K=12 p+12-60 t+84 u-6 x_{1}-42 x_{2}-42 x_{3}+42 x_{4}, \\
& 588 L=12 p+12+24 t+8 x_{1}+98 x_{5}+294 x_{6} .
\end{aligned}
$$

3. An application. It is well known (see for example [11, p. 26]) that 2 is a seventh power $(\bmod p)$ if and only if $(0,0) \equiv 1(\bmod 2)$, that is by the Theorem if and only if $x_{1} \equiv 0(\bmod 2)$. Note that $x_{1} \equiv 1(\bmod 7)$ is given uniquely by the system (1.2)-(1.4). For further results of this kind see [8].

\section{REFERENCES}

1. L. D. Baumert and H. Fredricksen, The cyclotomic numbers of order eighteen with applications to difference sets, Math. Comp. 21 (1967), 204-219. MR 36 \#6370.

2. L. E. Dickson, Cyclotomy, higher congruences, and Waring's problem. I, II, Amer. J. Math. 57 (1935), 391-424, 463-474.

3. - Cyclotomy and trinomial congruences, Trans. Amer. Math. Soc. 37 (1935), 363-380.

4. - Cyclotomy when $e$ is composite, Trans. Amer. Math. Soc. 38 (1935), $187-200$.

5. E. Lehmer, On the quintic character of 2, Bull. Amer. Math. Soc. 55 (1949), 62-63. Abstract $\sharp 72$. 
6. E. Lehmer, On the number of solutions of $u^{k}+D \equiv w^{2}(\bmod p)$, Pacific J. Math. 5 (1955), 103-118. MR 16, 798.

7. P. A. Leonard and K. S. Williams, A diophantine system of Dickson, Rend. Accad. Naz. Lincei 56 (1974), 145-150.

8. - The septic character of 2, 3, 5, and 7, Pacific J. Math. 52 (1974), $143-147$.

9. J. B. Muskat, The cyclotomic numbers of order fourteen, Acta Arith. 11 (1965/66), 263-279. MR $33 \# 1302$.

10. J. B. Muskat and A. L. Whiteman, The cyclotomic numbers of order twenty, Acta Arith. 17 (1970), 185-216. MR $42 \# 3050$.

11. T. Storer, Cyclotomy and difference sets, Lectures on Advanced Math., No. 2, Markham, Chicago, Ill., 1967. MR $36 \# 128$.

12. A. L. Whiteman, Theorems on quadratic partitions, Proc. Nat. Acad. Sci. U.S.A. 36 (1950), 60-65. MR 11, 332.

13. - The cyclotomic numbers of order sixteen, Trans. Amer. Math. Soc. 86 (1957), 401-413. MR 19, 1160.

14. The cyclotomic numbers of order twelve, Acta Arith. 6 (1960), 5376. MR $22 \sharp 9480$.

15. - The cyclotomic numbers of order ten, Proc. Sympos. Appl. Math., vol. 10, Amer. Math. Soc., Providence, R.I., 1960, Pp. 95-111. MR $22 \sharp 4682$.

DEPARTMENT OF MATHEMATICS, ARIZONA STATE UNIVERSITY, TEMPE, ARIZONA 85281

DEPARTMENT OF MATHEMATICS, CARLETON UNIVERSITY, OTTAWA, ONTARIO, K1S SB6, CANADA (Current address of both authors) 